\title{
Cramér-Rao Lower Bound Analysis for Many-to-One UAV Tracking Using FDOA Measurements
}

\author{
Byoung-Yoon Min, Chan-ho An, Dong-Ku Kim, Seok Jun Hong, Jin Sang Jang, and Ui Young Pak
}

\begin{abstract}
This paper considers the application for tracking of one mobile emitter using a sequence of frequency difference of arrival (FDOA) measurements obtained by single pair of receivers at a time. We suppose there is only one unmanned aerial vehicle (UAV) as emitter without data link relation problems, such as disconnection of data link or false measurements. Each FDOA measurement defines a region of possible emitter locations around a unique hyperbola. These analysis leads to the Cramér-Rao lower bound (CRLB) for estimation of movements. Through the analysis we propose the manner for tracking through the error-ellipsoids acquired from each CRLB. Since the performance measure is the length of error ellipsoid axes, we compare the effects of various environments.
\end{abstract}

Index Terms-Frequency difference of arrival (FDOA), Cramér-Rao lower bound (CRLB), unmanned aerial vehicle (UAV), tracking, ground station (GS)

\section{INTRODUCTION}

Many tracking researches has developed with highly accurate algorithm by comparing certain movement and measurement models and diminishing the noise effects from a high fidelity, expensive sensor. In these days, the trend has gone toward utilization of a number of cheap sensors [1].

The unmanned aerial vehicles (UAVs) technology has been one of the important examples of tracking. Since most emitter on the surface of the ground has enabled many applications, both military surveillance and civilian activity, this technology needs better visibility [2]. The UAVs use small omni/directional antennas and measure time and frequency of arrival of a pair of signals at the sensors. A single measurement of arrival type is not utilized to acquire geographical information. When two or more receivers take the same signal, time difference of arrival (TDOA) or frequency difference of arrival (FDOA) can be obtained. With these measurements, the emitter location can be estimated from the intersection of two or more hyperbolas [3]. There is another approach that use two moving emitter with

Manuscript received September 15, 2012; revised October 12, 2012.This work was supported in part by the Samsung Thales Co., Ltd. in Republic of Korea. This work was also supported by Agency for Defense Development and Samsung Thales, Co., Ltd. in Republic of Korea. Cramér-Rao Lower Bound Analysis for Many-to-One UAV Tracking Using FDOA Measurements, Byoung-Yoon Min, and Chan-ho An, Dong-Ku Kin, Seok Jun Hong, Jin Sang Jang, and Ui Young Pak.

B. Y. Min, C. An, and D. K. Kim are with the school of Electrical Electronic Engineering, Yonsei university, Seoul, Korea (e-mail: minby33@yonsei.ac.kr, chanhoa@yonsei.ac.kr, dkkim@yonsei.ac.kr)

S. J. Hong is with the Samsung Thales Co., Ltd, Seongnam-City, Gyeonggi-Do, Korea (e-mail: sj123.hong@samsung.com )

J. S. Jang and U. Y. Pak are with the Agency for Defense Development, Dajeon, Korea (e-mail: jangjs@add.re.kr, puy@add.re.kr ).
TDOA measurements [4].

The FDOA and TDOA measurements are utilized jointly estimation from communication signals. Narrowband signals have better FDOA measurements; otherwise wideband signals have better TDOA measurements. Thus their combination has both advantages and these results are noticeable [5]. Estimating the location of an emitter from a combination of TDOA and FDOA measurements is a highly nonlinear problem. This measurement obtained by a number of sensors is used in an over-determined situation to estimate instantaneous position and velocity of the emitter [6]-[7].

We consider a certain system in which we have many receivers, and these are not moving. On the other hand, the emitter is moving then we want to estimate the location of UAV with FDOA measurements. From many receivers, the diversity of FDOA increase then we obtain precise performance.

This paper is organized as follows. In section 2, the theoretical backgrounds are introduced consequently. In each of backgrounds, we suggest the research method and key-point to analyze the performance. Section 3 discusses simulation parameters, experiments conditions, and results. In the end, we conclude this paper in section 4.

\section{THEORETICAL BACKGROUNDS}

A moving UAV on the atmosphere of the earth needs to be tracked by ground station (GS) on the certain surveillance. In this paper we assume certain flat ground and GSs are located in this ground. It is quite uncomplicated to consider the geometry without any geographical conditions. The signal model of Doppler and delay is divided into two parts: signal of transmitter and receiver. The analytic signals model of transmitter $\tilde{s}_{t}(t)$ and receiver $\tilde{s}_{r}(t)$ is

$$
\begin{gathered}
\tilde{s}_{t}(t)=E(t) e^{j\left[\omega_{c} t+\phi(t)\right]}, \\
\tilde{s}_{r}(t)=E\left([1-v / c] t-\tau_{d}\right) e^{j\left\{\omega_{c}\left([1-v / c] t-\tau_{d}\right)+\phi\left([1-v / c] t-\tau_{d}\right)\right\}}
\end{gathered}
$$

where $E(t)$ denotes the energy of signal at time $t, v$ is the relative velocity between emitter and receiver, $c$ is speed of light, $\tau_{d}$ is time-delay, and $\omega_{c}$ is carrier frequency. When we apply the narrowband approximation [8], receiver signal model is represented by

$$
\tilde{S}_{r}(t)=e^{-j \omega_{c} \tau_{d}} \times e^{-j \omega_{c}(v / c) t} \times e^{j \omega_{c} t} \times E\left(t-\tau_{d}\right) e^{j \phi\left(t-\tau_{d}\right)}
$$

There are four terms: constant phase term, Doppler shift term, carrier term, and transmitted signal's low-pass equivalent (LPE) term. Therefore estimator for FDOA is 
applied to complex ambiguity function (CAF), which represents cross-correlation surface of received signal. Although finding peak of CAF gives us Doppler and delay value of the network, we are not interested in details. In this paper these are basic assumptions for expressions of all problem settings.

\section{A. Frequency Difference of Arrival}

Most of moving emitters bring about the Doppler Effect due to their velocity. Relative movement between GS and UAVs shifts the signal received by the sensor in frequency domain. Assuming stationary GSs, the relative velocity of the UAV and the corresponding Doppler shift are given in each continuous time samples.

In this paper we assume that all network systems are pair-wise network. Fig. 1 depicts a many(GSs)-to-one(UAV) tracking model with FDOA. In the centralized network, there are one or some command centers which supervise whole data links. Due to signal-data in common, it passes through correlation effect that can be harmful for network service qualities. On the other hand, each measurement is acquired by pair of receiver instead of central command. All pairs of measurements are not equally good, however. We assume all GSs are apart from each neighbor pairs to preserve the fairness of Doppler shift measurements. The kinematic state of this network model of GSs, $\mathbf{X}_{G S}^{i}$, and UAV, $\mathbf{X}_{U A V}$ at time $k$ can be expressed in two-dimensional space to simplify a real model. Since GSs have fixed locations, time $k$ can be omitted. There are $2 N$ stationary GSs and single moving UAV in which,

$$
\begin{aligned}
& \mathbf{X}_{G S}^{i}=\left[\begin{array}{llll}
x_{G S}^{i} & y_{G S}^{i} & 0 & 0
\end{array}\right]^{T}, i=1, \ldots, 2 N, \\
& \mathbf{X}_{U A V}[k]=\left[\begin{array}{llll}
x[k] & y[k] & V_{x}[k] & \left.V_{y}[k]\right]^{T},
\end{array}\right.
\end{aligned}
$$

where $V_{x}, V_{y}$ are velocity of UAV in $\mathrm{x}$-axis and y-axis, respectively, and $N$ denotes the integer number of measure-ments pair, which increase when there are measurements from many GSs collected over time.

Therefore FDOA measurements of $i, j$ th GS pair is

$$
\begin{aligned}
\varsigma_{i, j}=\frac{f_{0}}{c} \frac{d}{d t}\left(R_{1}-\right. & \left.R_{2}\right)=\frac{f_{0}}{c}\left[\frac{\left(x-x_{G S}^{i}\right) V_{x}+\left(y-y_{G S}^{i}\right) V_{y}}{\sqrt{\left(x-x_{G S}^{i}\right)^{2}+\left(y-y_{G S}^{i}\right)^{2}}}\right. \\
& \left.-\frac{\left(x-x_{G S}^{j}\right) V_{x}+\left(y-y_{G S}^{j}\right) V_{y}}{\sqrt{\left(x-x_{G S}^{j}\right)^{2}+\left(y-y_{G S}^{j}\right)^{2}}}\right]
\end{aligned}
$$

where $f_{0}$ is the frequency of transmitted signal and $R_{i}$ is the range between $i$ th GS and the UAV. The time factor $k$ of UAV state model is abbreviated for simplicity.

\section{B. Measurement Error}

Contained by sufficiently large time and bandwidth, received signal and noise processes product thoroughly enough signal-to-noise ratio. The joint FDOA measurements estimation is diagonal. In other words, FDOA estimates are approximately uncorrelated.

Some of FDOA estimators approach to the CRLB with approximated hypothesis of non-correlation between pairs of measurements error. In this paper we assume that these errors are additive Gaussian with zero-mean and be taken by uncorrelated property. Through the results of (6), we can obtain the $2 N$-dimensional measurement data vector as follows,

$$
\mathbf{s}=\left[\begin{array}{lllll}
\varsigma_{1,2} & \varsigma_{2,3} & \cdots & \varsigma_{2 N-1,2 N} & \varsigma_{2 N, 1}
\end{array}\right]^{T}
$$

Each of these measurements has a mutually independent error with covariance $\mathbf{C}$. Let $\mathbf{r}_{i}$ is $i$ th received measurement,

$$
\mathbf{r}_{i}=\mathbf{s}_{i}+\boldsymbol{\varepsilon}_{i}
$$

where $\boldsymbol{\varepsilon}$ denotes error vector with normal distribution of $N\left(0, \sigma_{\varepsilon}^{2}\right)$. It can be expanded to the covariance matrix,

$$
\mathbf{C}=\operatorname{diag}\left\{\mathbf{C}_{1}, \mathbf{C}_{2}, \ldots, \mathbf{C}_{2 N}\right\}=\left[\begin{array}{ccc}
\mathbf{C}_{1} & \mathbf{0} & \mathbf{0} \\
\mathbf{0} & \ddots & \mathbf{0} \\
\mathbf{0} & \mathbf{0} & \mathbf{C}_{2 N}
\end{array}\right]
$$

where $\mathbf{C}_{k}$ denotes covariance of $k$ th measurement. If we gather more FDOA pair than fixed number of pair $2 N$ through many sample times, the size of $\mathbf{C}_{k}$ can be larger. In this paper we utilize this extension to achieve proper lower bound performance.

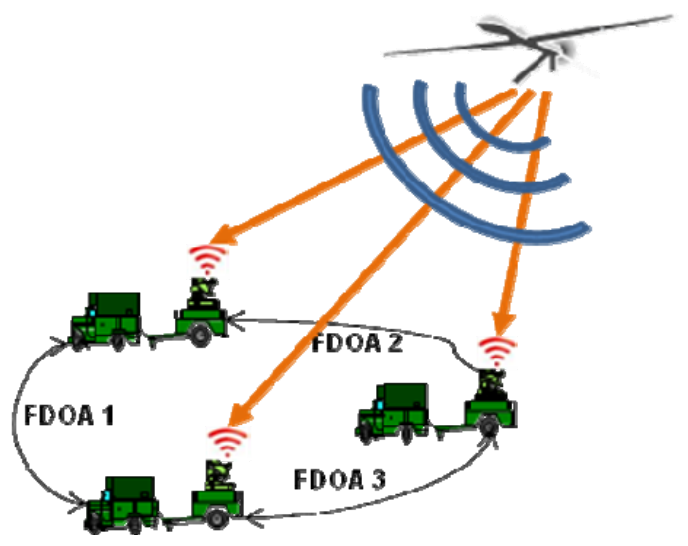

Fig. 1. Many(GSs)-to-one(UAV) system

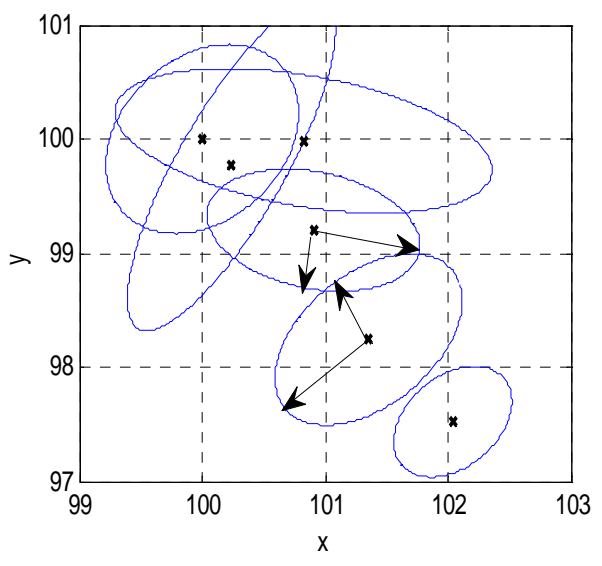

Fig. 2. CRLB bounds and their axes 


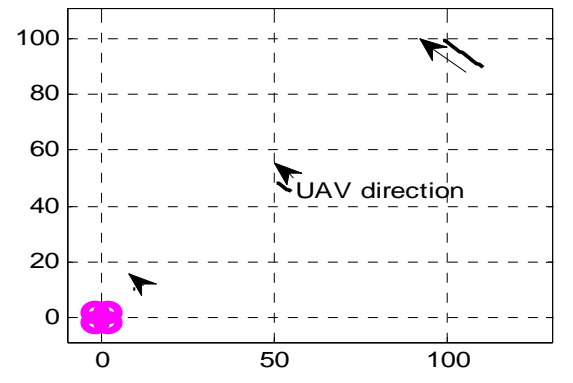

Fig. 3. Simulation model of UAV movements

\section{Cramér-Rao Lower Bound}

CRLB is a well-known lower bound on the variance of any unbiased estimator. Sometimes there is an easier method to find the CRLB from alternate form [9]. It is defined by Fisher information matrix (FIM), which is denominator in CRLB; it is a measure of the expected goodness of the data for the purpose of making estimation.

Since we have a deterministic signal plus Gaussian noise, CRLB depends on not parameters of variability of covariance but those of mean. The CRLB matrix is,

$$
\mathbf{C}_{\text {CRLB }}\left(\mathbf{x}_{e}\right)=\left[\mathbf{H}^{T} \mathbf{C}^{-1} \mathbf{H}\right]^{-1},
$$

where $\mathbf{x}_{e}$ denotes $\left[\begin{array}{llll}x & y & z & f_{0}\end{array}\right]^{T}$, and $\mathbf{H}$ denotes Jacobian matrix of measurements data vector $\mathbf{s}$,

$$
\mathbf{H} \triangleq \frac{\partial \mathbf{s}\left(\mathbf{x}_{e}\right)}{\partial \mathbf{x}_{e}}=\left[\begin{array}{llll}
\frac{\partial \mathbf{s}\left(\mathbf{x}_{e}\right)}{\partial x} & \frac{\partial \mathbf{s}\left(\mathbf{x}_{e}\right)}{\partial y} & \frac{\partial \mathbf{s}\left(\mathbf{x}_{e}\right)}{\partial z} & \frac{\partial \mathbf{s}\left(\mathbf{x}_{e}\right)}{\partial f_{0}}
\end{array}\right] .
$$

In this paper we consider only two-dimension, so four-dimensional CRLB from (10) is projected in two-dimension space.

Given CRLB form, we acquire error ellipsoids to observe the performance of lower bound. If we suppose that whole past estimations are perfect, we can assume the state of UAV in two-dimensional vector $\boldsymbol{\theta}$,

$$
\boldsymbol{\theta}=\left[\begin{array}{ll}
x & y
\end{array}\right]^{T},
$$

and define the equi-height contours such as ellipsoids

$$
\boldsymbol{\theta}^{T} \mathbf{C}_{C R L B}^{-1} \boldsymbol{\theta}=-2 \ln \left(1-P_{e}\right)=k,
$$

where $P_{e}$ denotes the probability that the estimation points will lie inside the ellipse. Since it is important to measure the length of ellipsoid, we can get from Eigen-value-decomposition. In Fig. 2, the eigenvectors denote the principle axes of ellipsoids and the eigenvalues denote the length of principle axes. Therefore we apply these factors for tracking when UAV have no information for itself.

\section{SIMULATION EXPERIMENTS}

\section{A. Movement Model of UAVs}

Above the earth, the UAVs go around certain course to fulfill official duties. In this paper we consider linear movements when UAV is affected by some other flight conditions: wind, rain, inexperienced control, etc. When we determine these conditions represented as zero mean white Gaussian vectors, the state model is

$$
\mathbf{X}_{U A V}[k+1]=A \cdot \mathbf{X}_{U A V}[k]+\boldsymbol{\eta}
$$

where $A$ denotes state transition matrix of position and velocity, and $\boldsymbol{\eta}$ denotes the Gaussian error vector,

$$
A=\left[\begin{array}{llll}
1 & 0 & T & 0 \\
0 & 1 & 0 & T \\
0 & 0 & 1 & 0 \\
0 & 0 & 0 & 1
\end{array}\right], \boldsymbol{\eta}=\left[\begin{array}{c}
0 \\
0 \\
\eta_{x} \\
\eta_{y}
\end{array}\right]
$$

where $\eta_{x}, \eta_{y}$ have normal distribution of $N\left(0, \sigma_{\eta_{x}}^{2}\right)$, and $N\left(0, \sigma_{\eta_{y}}^{2}\right)$, respectively. This model is well-approached for UAV working conditions because UAV does not have change of movement direction or speed dramatically. In Fig. 3 , four circles denote the GSs, and three black paths are UAV movements. There are three different flights to analyze performance with distance parameter.

\section{B. Simulation Environments}

Here we consider some factors for simulation. The speed of UAV is set to about $120 \mathrm{~km} / \mathrm{h}$ and direction of the speed is fixed although it has a little variation due to the error vector $\boldsymbol{\eta}$. About geolocation of GSs, we consider 4 with equal distance from neighbor. Each of them has $( \pm 2, \pm 2) \mathrm{km}$. In this assumption, we take $4 T$ different measurements where $T$ denotes period to gather enough data measurements. The value of $T$ is a comparison factor for cumulative density function (CDF) of error ellipsoid. Assuming one time sample is $0.05 \mathrm{sec}$, and $T=(20,40,80)$, tracking interval is set to $(1,2,4)$ sec. The carrier frequency of signal is $f_{0}=15 \mathrm{GHz}$, and $P_{e}$ for error ellipsoids is set to 0.9. The variance of two Gaussian vectors; measurement noise and movement noise, denoted by $\sigma_{\varepsilon}^{2}, \sigma_{\eta}^{2}$. Supposed by conditions of nature, $\sigma_{\eta_{x}}^{2}=\sigma_{\eta_{y}}^{2}=1.8 \mathrm{~km} / \mathrm{h}$, and $\sigma_{\varepsilon}^{2}$ is $5 \%$ root-mean-square (RMS) value of measured FDOA in tracking interval.

\section{Simulation Results}

In all results we obtained the length of ellipsoid axes. Since the number of axes of ellipsoids is two, we defined $R_{\max }$ as one of maximum length of two. There are the observations for distribution of $R_{\max }$ in Fig. 4. The bound performance is better if the CDF exists in left direction. At first, if we gather more FDOA measurements over time interval, these performances of CDF gets better. Intuitively, it depends on the size of measurements data matrix, which contains uncorrelated measurements pair.

Furthermore the distance between GSs and UAV is the important parameter. $R_{\max }$ does not change linearly with distance. It is not intuitive results as we can see the arrow of 
“Max 1deg error.” By the degree made from GSs to ellipsoid axis, we can measure how large these CRLB relatively. If the emitter is near to GSs, these CRLB is decreasing more sharply than linear decreasing. We can calculate this relation through the tangent formation.

Since the relative distance between GSs and UAV highly affects to CRLB, the flight time of UAV is variable linearly with distance. The RMS value of FDOA measurements are calculated at the receiver per tracking interval, the variance of measurements noise is not fixed.

\section{CONCLUSION}

This paper presents analysis for estimation based on integrating data from FDOA measurements over time. These measurements are obtained at each tracking interval of the art. At this interval, CRLB represents the bound of UAV location with gathered measurements over time. From the eigenvalues and eigenvectors, an efficient method is proposed through the difference simulation experiments.

Simulation results have shown that proposed bound technique gives us degree of error by tangent function. When GSs use sharp beamforming, we can apply these factors directly. Moreover, from measurement fusion except for FDOA, can expect more precise estimation through the CRLB.

\section{ACKNOWLEDGMENT}

This work was supported by Samsung Thales, Co., Ltd. in Republic of Korea.

This work was also supported by Agency for Defense Development and Samsung Thales, Co., Ltd. in Republic of Korea.

\section{REFERENCES}

[1] F. Fletcher, B. Ristic, and D. Musicki, "Recursive estimation of emitter location using TDOA measurements from two UAVs," in 10th Int Conf. Inf. Fusion, 2007.

[2] C. H. An, J. Yang, D. K. Kim, U. Y Pak, and K. Kwon, "Numerical Analysis of Transmission Efficiency to Guarantee the QoS for Densely Distributed UAV with Randomly Located Ground Station,” in Proc. IEEE ICCAE'10, vol. 2, pp. 426-429, Feb. 2010

[3] K. Ho and Y. Chan, "Solution and performance analysis of geolocation by TDOA,” IEEE Trans. Aerosp. Electron. Syst., vol. 29, no. 4, pp. 1311-1322, Oct. 1993.

[4] F. Fletcher, B. Ristic, and D. Mušicki, "TDOA measurements form two UAVs,” in 10th Int. Conf. Inf. Fusion, Quebec, QC, Canada, Jul. 2007.

[5] D. Mušicki, R. Kaune, and W. Koch, "Mobile emitter geolocation and tracking using TDOA and FDOA measurements," IEEE Trans. on Signal Process, vol. 58, no. 3, Mar. 2010.

[6] K. C. Ho, X. Lu, and L. Kovavisaruch, "Source localization using TDOA and FDOA measurements in the presence of reciever location errors: Analysis and solution,” IEEE Trans. Signal Process, vol. 55, no. 2, pp. 684-696, Feb. 2007.

[7] K. C. Ho and Y. T. Chan, "Geolocation of a known altitude object from TDOA and FDOA measurements," IEEE Trans. Aerosp. Electron. Syst., vol. AES-18, no. 2, pp. 214-218, Mar. 1982

[8] S. M. Kay, "Fundamentals of Statistical Signal Processing: Estimation Theory,” Prentice-Hall, Inc., Upper Saddle River, NJ, USA, 1993.

[9] W. Gardner, "Likelihood sensitivity and the Cramér-Rao bound (Corresp.)," IEEE Trans. Info. Theory, vol. 25, no. 4, pp. 491, Jul 1979.

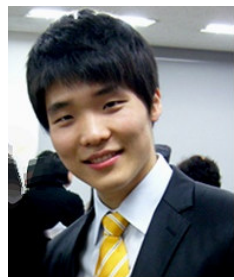

Byoung-Yoon Min received the B.S degree in electrical and electronic engineering from Yonsei University, Seoul, Korea, in 2010. He is currently a candidate for integrated M.S and Ph.D degree at Yonsei University.

His main research interests are virtual MIMO techniques, Hierarchical cooperation, and mobile relaying transmission. Also He was working on the project for Agency Defense Development (ADD) to analyze and simulate UAV tracking researches.

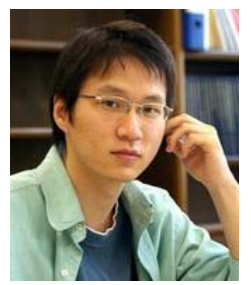

Chan-ho An was born in Seoul, Korea, in 1983. He received the B.S degree in radio communication engineering from Kyunghee University, Suwon, Korea in 2006 and M. S. degree in electrical and electronic engineering from Yonsei University, Seoul, Korea in 2008. He is currently working towards his Ph.D degree at Yonsei University.

His main research interests are communication signal processing, MIMO transceiver scheme, OFDMA/SC-FDMA, lattice reduction, MIMO Radar and UAV tracking technologies.

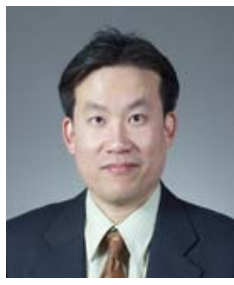

Dong-Ku Kim received the B. Eng degree from Hankook Aviation university, Korea, in 1983 and the M. Eng and Ph.D degree from the university of South California, Los Angeles, in 1985 and 1992, respectively. He was a research Engineering with the cellular infrastructure group, Motorola, from 1992 to 1994. Since 1994, he has been with Electrical and Electronic Engineering Department, Yonsei University, Seoul, where he is currently a Professor. His main research interests are 4G communication systems, cooperative MIMO, wireless mobile relay, compressive sensing, and energy efficient wireless communication technologies. He was a Director of Qualcomm Yonsei CDMA Research Lab and Deputy Director of the Radio Communication Research Center, Yonsei University. He is currently Chief Editor for Transaction on Telecommunication of the Korea Institute of Electronic Engineers.

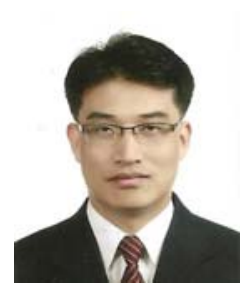

Seok Jun Hong received the B.S degree in electronic engineering from KwangWoon University, Korea, and the M.S degree in electrical and electronic engineering from Yonsei University, Seoul, Korea.

His main research interests are Ultra High frequency techniques, antenna technologies, and wireless communications. He is currently working on the Samsung Thales, Co., Ltd. as a senior researcher.

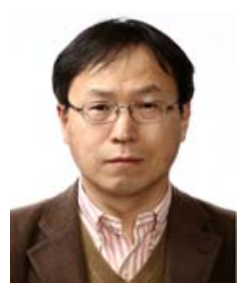

Jin Sang Jang received the B.S and M.S degrees in electircal enginnering from SeongGyunGwan University, Korea, and the Ph.D degree in electronic computer science from University of Toledo, USA.

His main research interests are MIMO data links, OFDM, and communication networks. $\mathrm{He}$ is currently working on the Agency Defense Development (ADD) as a senior researcher.

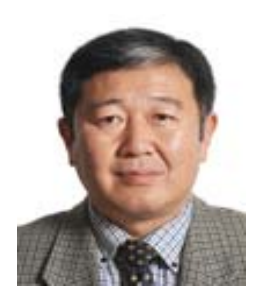

Ui Young Pak received the Ph.D degree in electrical and electronic engineering from Yonsei University, Seoul, Korea.

His main research interests are data links and mobile communication. He is currently working on the Agency Defense Development (ADD) as a senior researcher. 